\title{
Electronic structure of the contact between carbon nanotube and metal electrodes
}

S. Dag, O. Gülseren, S. Ciraci, and T. Yildirim

Citation: Appl. Phys. Lett. 83, 3180 (2003); doi: 10.1063/1.1616662

View online: http://dx.doi.org/10.1063/1.1616662

View Table of Contents: http://aip.scitation.org/toc/apl/83/15

Published by the American Institute of Physics

\section{Articles you may be interested in}

Effects of local structural defects on the electron transport in a carbon nanotube between Cu electrodes Applied Physics Letters 97, 242112 (2010); 10.1063/1.3527918

First-principles study of contact between Ti surface and semiconducting carbon nanotube Journal of Applied Physics 102, 013709 (2007); 10.1063/1.2748716

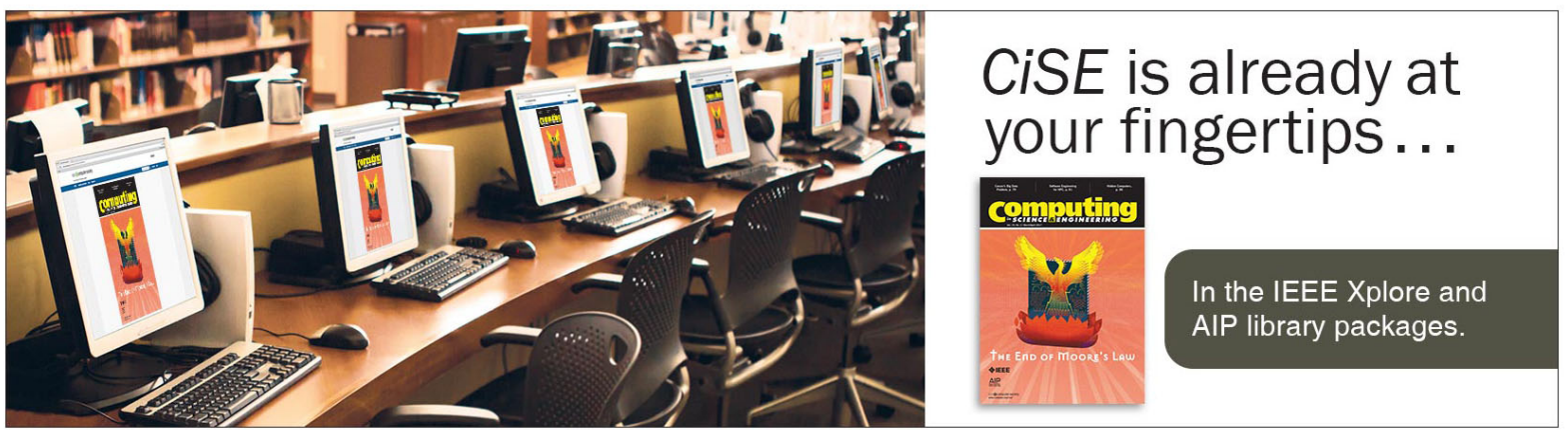




\title{
Electronic structure of the contact between carbon nanotube and metal electrodes
}

\author{
S. Dag, O. Gülseren, and S. Ciracia \\ Department of Physics, Bilkent University, Ankara 06800, Turkey
}

T. Yildirim

NIST Center for Neutron Research, National Institute of Standards and Technology, Gaithersburg, Maryland 20899

(Received 4 June 2003; accepted 12 August 2003)

\begin{abstract}
Our first-principles study of the contact between a semiconducting single-walled carbon nanotube (s-SWNT) and metal electrodes shows that the electronic structure and potential depend strongly on the type of metal. The s-SWNT is weakly side-bonded to the gold surface with minute charge rearrangement and remains semiconducting. A finite potential barrier forms at the contact region. In contrast, the molybdenum surface forms strong bonds, resulting in significant charge transfer and metallicity at the contact. The radial deformation of the tube lowers the potential barrier at the contact and increases the state density at the Fermi level. (C) 2003 American Institute of Physics. [DOI: $10.1063 / 1.1616662]$
\end{abstract}

Nanoscience and nanotechnology have opened a new frontier aiming at the ultimate miniaturization of electronic circuits with ultrahigh speed and high-density components presenting new functionalities. The switching of the current in a semiconducting single-walled carbon nanotube (sSWNT) at room temperature by an external electric field has been utilized to fabricate new field-effect transistors (FET). ${ }^{1-3}$ The interaction between s-SWNT and metal electrode, and the Schottky barrier, $\Phi_{B}$ formed thereof have been proposed as the origin of FET operation. Model calculations have been carried out to provide further understanding of experimental $I-V$ characteristics. ${ }^{4,5}$ The SWNT-electrode interaction and resulting electronic structure are crucial for the electron transport and hence for all device properties. This letter presents an analysis of the self-consistent-field (SCF) electronic potential and electronic energy structure of a zigzag $(8,0)$ SWNT side-bonded to either gold or molybdenum electrodes.

Calculations are performed using first-principles pseudopotential plane wave method ${ }^{6}$ within the density functional theory and the generalized gradient approximation. ${ }^{7}$ A metal electrode is presented by a slab consisting of five atomic planes. First, the lattice parameters of the slab have been changed slightly to match to that of the SWNT. Induced strains along the directions perpendicular to the axis of SWNT have been determined by using bulk Poisson's ratio. The calculated stress is then minimized by varying the lattice parameters of the SWNT-adsorbed slab. Finally, all atomic positions, except those at the bottom two planes of slab, have been fully relaxed. The lattice parameter of the $\mathrm{Au}$ (Mo) substrate along the SWNT axis expanded (contracted) by $1.5 \%(2.7 \%)$ upon relaxation. Mo atoms in the close proximity of SWNT have significant perpendicular displacements. In the study of the effect of radial deformation, the SWNT is pressed between two metal electrodes, each made of three

${ }^{a)}$ Electronic mail: ciraci@fen.bilkent.edu.tr atomic planes having fixed atomic positions and twodimensional periodicity lattice-matched to the tube. Note that the supercell geometry gives rise to a continuous contact of infinite extension, but it can provide accurate prediction of the electronic structure and atomic positions, which are crucial to determine the Fermi level pinning and band diagram of finite semiconductor-metal heterostructures.

We start by summarizing our results for the adsorption of individual $\mathrm{Au}$ and $\mathrm{Mo}$ atoms, since the character of their bonding determines the interaction between SWNT and corresponding metal electrodes. ${ }^{8}$ The adsorption geometry is determined first by placing single Au or Mo atom at different possible adsorption sites (i.e., above the center of hexagon, $\mathrm{H}$-site; just above a carbon atom, T-site; and above the axial or zigzag $\mathrm{C}-\mathrm{C}$ bond, $\mathrm{A}-$ and $\mathrm{Z}$-sites) and then by optimizing the atomic structures. We found that the binding energy of an individual $\mathrm{Au}$ atom is, in general, weak and ranges between 0.3 and $0.5 \mathrm{eV}$ depending on the adsorption site. In contrast, the bonding of an individual $\mathrm{Mo}$ atom at the $\mathrm{H}$-site is rather strong and involves significant charge transfer.

The contact on the gold electrode is formed by placing the s-SWNT on the $\mathrm{Au}(100)$ surface, so that a surface $\mathrm{Au}$ atom faces the center of the hexagon. The $\mathrm{Au}-\mathrm{SWNT}$ distance, $s$, was initially $2.2 \AA$, but it increased to $3.18 \AA$ as a result of full structure optimization. The interaction energy $E_{i}$ is calculated by subtracting the total energies of SWNT alone and metal electrode alone from the total energy of the electrode + SWNT system. For the Au, we found $E_{i}$ to be very small and repulsive $(-48 \mathrm{meV}$ per supercell). The equilibrium position may correspond to a local shallow minimum, but it shall be further stabilized by Van der Waals interaction. This situation implies a weak $\mathrm{Au}-\mathrm{SWNT}$ interaction which is characterized by physisorption.

The effect of the Au electrode on the band structure of the bare SWNT may be deduced from the single Au atom adsorbed on the SWNT. Adsorbed single Au does not induce any significant change in the bands of the bare tube; the form of the band gap remains unaltered. Au gives rise to a single 

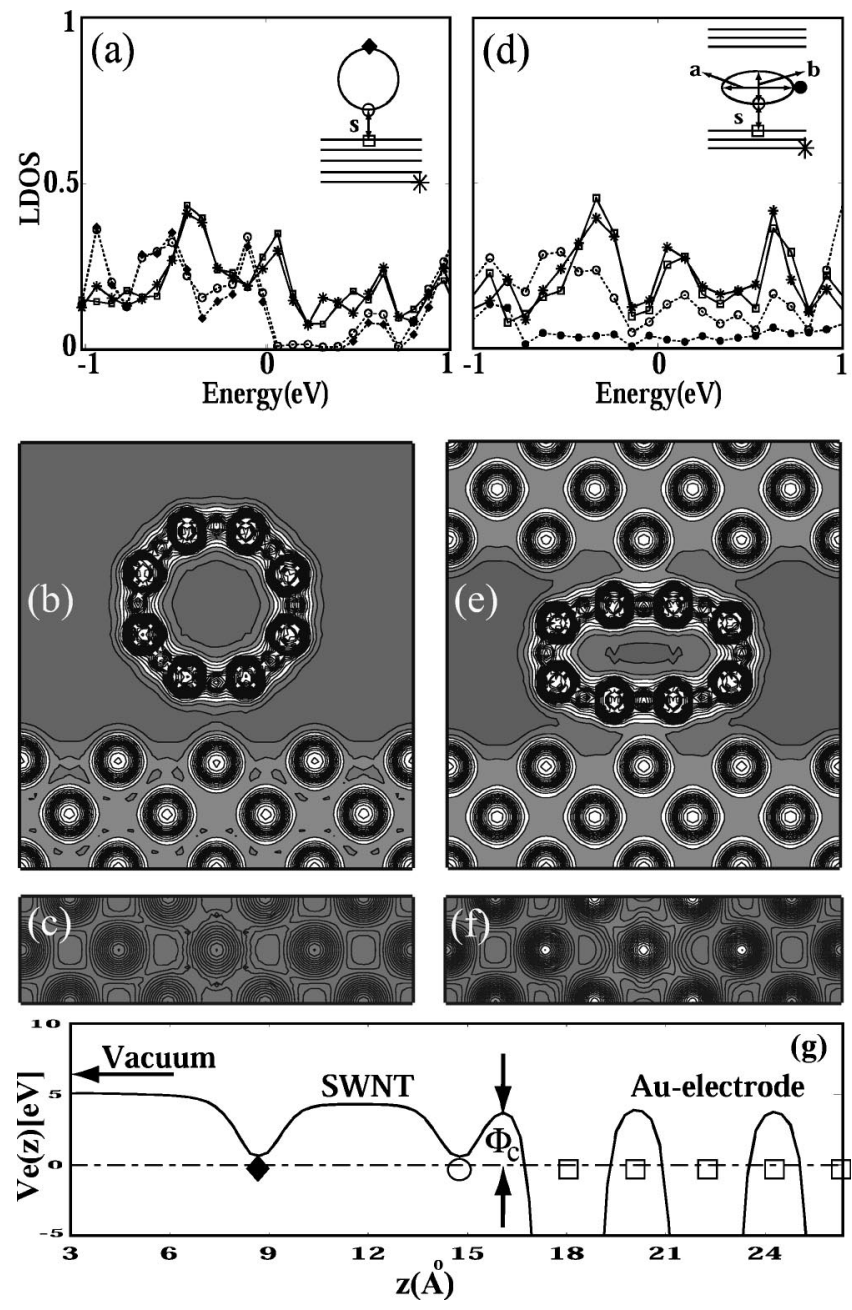

FIG. 1. (a) LDOS at different $\mathrm{Au}$ and $\mathrm{C}$ atoms of the $(8,0)$ zigzag SWNT side-bonded to the $\mathrm{Au}(100)$ surface. The location of atoms are described by inset. The zero of energy is taken at the Fermi energy $E_{\mathrm{F}}$. State densities shown by empty circle and filled diamonds correspond to six neighboring carbon atoms. Other densities are for single atoms. (b) Contour plots of the SCF electronic potential, $V_{e}(r)$, on a vertical plane. (c) Same as in (b) on a horizontal plane bisecting $s$. In the dark gray regions $V_{e}(r)>E_{\mathrm{F}}$. (d), (e), and (f) correspond to the radially deformed $(8,0)$ SWNT pressed between two three-layer $\mathrm{Au}(100)$ slabs with $b / a=0.47$ as shown by inset. State densities shown by empty circles in (d) correspond to six neighboring carbon atoms. Other densities are for single atoms. (g) Variation of $V_{e}(z)$ on a perpendicular line passing through the center of SWNT.

isolated band. The effect on the electronic structure can be revealed for the local density of state (LDOS) calculations. The LDOS calculated for two Au atoms at different sites (one is at the contact just below the SWNT hexagon; the second being farthest to SWNT mimics the clean Au surface) in Fig. 1(a) have finite state density at the Fermi level. These two LDOS are similar-there are only minute changes; whereas the LDOS of the carbon atoms at the contact still has a band gap. The Fermi level lies near the top of the valence band of SWNT and complies with a small $\Phi_{B}$ in the hole-doping picture. The LDOS at the carbon atom which is farthest from the metal electrode displays a state distribution similar to that of the carbon atom at the contact region. Calculated total charge density of SWNT bonded to the metal electrode, E; that is, $\rho[\mathrm{SWNT}+\mathrm{E}]$, and difference charge density, $\quad \Delta \rho=\rho[\mathrm{SWNT}+\mathrm{E}]-\rho[\mathrm{E}]-\rho[\mathrm{SWNT}], \quad$ shows minute charge rearrangement. These results indicate that the weak Au-electrode-SWNT interaction does not induce sig- nificant changes in the electronic structure. The SCF electronic potential between SWNT and Au electrode, $V_{e}(r)$ $=V_{\text {ion }}(r)+V_{H}(r)+V_{x}(r)$, is presented on a vertical plane and also on a horizontal plane bisecting $s$ in Figs. 1(a) and 1(b). The shaded area shows that the electronic potential energy at the contact yields a potential barrier $\Phi_{c}=V_{e}-E_{\mathrm{F}}$ $>0$. At the contact midway between the SWNT and the $\mathrm{Au}(100)$ surface $\Phi_{c}$ is calculated to be $\sim 3.9 \mathrm{eV}$, that is comparable with the calculated work function $(\Phi \sim 5 \mathrm{eV})$ of the Au slab. Figure $1(\mathrm{~g})$ shows the variation of $V_{e}(z)$ on a line passing through the center of the SWNT and perpendicular to the $\mathrm{Au}(100)$ surface. The effective potential barrier $\Phi_{c \text {,eff }}$ can be even higher owing to the increased confinement of electrons at the contact region. Therefore, electrons that are transferred from the metal to the semiconducting SWNT has to tunnel a potential barrier $\Phi_{c, \text { eff }}(r)$.

By pressing the s-SWNT between two Au slabs, hence by imposing a radial deformation on the tube [see the inset in Fig 1(d)], we examined the electronic structure and the contact potential. Normally, the semiconducting tubes undergo an insulator-metal transition, since the $\pi^{*}$-singlet states in the conduction band are lowered because of increased $\pi^{*}$ $-\sigma^{*}$ hybridization at the high curvature site and eventually overlap with the valence band. ${ }^{9-11}$ In the present case, at a radial deformation, $\epsilon_{r}=b / a=0.47$, the state density of SWNT+electrode system near $E_{\mathrm{F}}$ has increased partly due to increased state density of the metallized s-SWNT. The spacing $s$ has decreased to $2.6 \AA$, and eventually potential barrier has collapsed (i.e., $V_{e}<E_{\mathrm{F}}$ ) at specific sites at the contact [see Figs. 1(e) and 1(f)]. Under these circumstances, the electron from the SWNT can be ballistically transferred to the gold electrodes.

This situation is, however, different in the case of s-SWNT side-bonded to the Mo(110) surface, as shown in Fig. 2(a). Upon relaxation, the tube has rotated slightly so that $\mathrm{C}$ atoms tend to approach to Mo atoms. The interaction energy $E_{i}$ has been calculated to be $\sim 3.5 \mathrm{eV}$. The LDOS at the Mo atom interacting with the $\mathrm{C}$ atoms of the SWNT is different from that at the clean Mo(110) surface. Moreover, the LDOS at the carbon atom closest to the Mo surface has a finite state density at $E_{\mathrm{F}}$. In particular, the peak near $E_{\mathrm{F}}$ is associated with the $\mathrm{C}-\mathrm{Mo}$ bond states and hence may be identified as the metal-induced gap states (MIGS). The LDOS of the $\mathrm{C}$ atoms farthest from the contact has a band gap near $E_{\mathrm{F}}$. This situation indicates that the site of SWNT forming contact is conducting, while the opposite site farthest from the contact remains semiconducting. Owing to the strong Mo-C bond, the spacing between SWNT and Mo electrode is smaller $(s=1.96 \AA)$ than that with the Au electrode. The strong $\mathrm{C}-\mathrm{Mo}$ bond formation is clarified by the calculated total SCF charge density in Fig. 2(b). The difference charge density $\Delta \rho$ presented on a vertical plane in Fig. 2(c) shows the strong charge transfer to the $\mathrm{C}-\mathrm{Mo}$ bonds. The electronic potential calculated on the horizontal plane bisecting $s$ and also on a line from the SWNT to the Mo(110) surface through the contact show that $V_{e}(r)<E_{\mathrm{F}}$ at the contact region. Accordingly, a potential barrier $\Phi_{c}$ does not form at the extended contact between the SWNT-Mo electrode. The radial deformation on the s-SWNT induced by squeezing it between two $\mathrm{Mo}(110)$ electrodes gives rise to the met- 

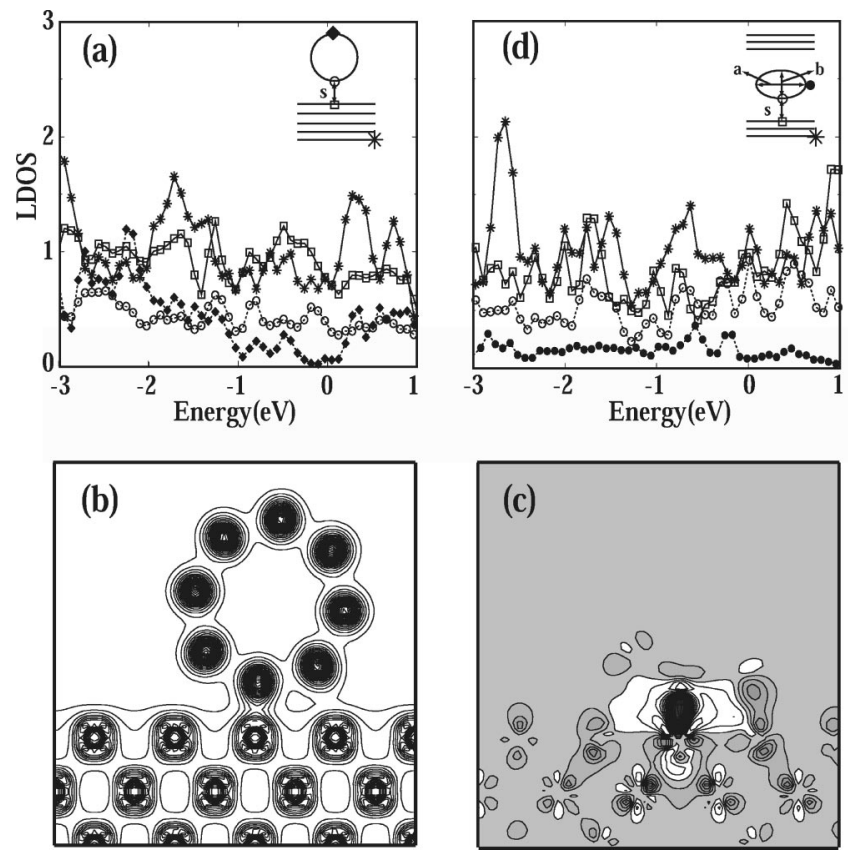

FIG. 2. (a) LDOS at different Mo and C atoms of the $(8,0)$ zigzag SWNT side-bonded to the Mo(110) surface. The location of atoms are described by inset. The zero of energy is taken at the $E_{\mathrm{F}}$. State densities shown by empty circles and filled diamond correspond to eight neighboring carbon atoms, Others are for single atoms. (b) Contour plots of the total SCF charge density on a vertical plane. (c) Difference charge density showing the charge depleted (white) regions and charge accumulate (black) regions. (d) LDOS corresponding to the $(8,0)$ SWNT radially deformed between two three-layer Mo(110) slabs with $b / a=0.56$ as shown by the inset. State densities shown by empty circles correspond to 10 neighboring carbon atoms.

allization of the tube and hence to the increase of state density at $E_{\mathrm{F}}$.

It appears that these results distinguish two different types of electronic structure for two different electrodes. A large spacing and sizable potential barrier between the tube and metal electrode, $\Phi_{c} \sim 3.9 \mathrm{eV}$, are characteristics of the contact made with the Au surface. This explains why the devices made from $\mathrm{Au}$ electrodes have high contact resistance. Here, weak coupling of electronic states cannot lead to MIGS, and the $(8,0)$ SWNT is identified to be semiconducting even after the contact has been set with the Au surface. Because of weak coupling and hence finite $\Phi_{c}$, the $\mathrm{s}$-SWNT-Au contact is reminiscent of the metal-oxidesemiconductor junction. A small $\Phi_{B}$ is estimated for the $p$-type character. Upon radial deformation, $s$ decreases and eventually $\Phi_{c}$ collapses. Similar features have been observed recently in scanning tunneling microscopy studies using a multiwalled carbon nanotube tip and $\mathrm{Au}(111)$ sample. $^{12}$ However, $\Phi_{c}$ has disappeared due to strong coupling between the states of s-SWNT and $\operatorname{Mo}(110) .{ }^{13}$ The height of the Schottky barrier $\Phi_{B}$ that forms at the SWNT-Mo(110) junction having a finite contact region followed by a free s-SWNT depends on the position where the Fermi level is pinned in the gap of s-SWNT. A crude estimate based on the LDOS suggests that $\Phi_{B} \sim 0.4 \mathrm{eV}$ for the $p$-type character. The small cross section of the tube does not allow us to identify a band diagram across the diameter. However, the bands are normally bent along the axis of the s-SWNT and the height of the barrier is monitored by the applied gate voltage. Finally, we note that the electronic properties of present metal-SWNT junctions, in particular the Fermi level pinning exhibits marked differences from those of metal-Si heterostructures, which are known to be insensitive to the type of metal.

This work was partially supported by the National Science Foundation under Grant No. INT01-15021 and TÜBÍTAK under Grant No. TBAG-U/13(101T010). One of the authors (S.C.) acknowledges partial support from Academy of Science of Turkey.

${ }^{1}$ S. J. Tans, A. R. M. Verscheuren, and C. Dekker, Nature (London) 393, 49 (1998); A. Bachtold, P. Hadley, T. Nakanishi, and C. Dekker, Science 294, 1317 (2001); T. Nakanishi, A. Bachtold, and C. Dekker, Phys. Rev. B 66, 073307 (2002).

${ }^{2}$ H. T. Soh, C. Quate, A. F. Morpurgo, C. M. Marcus, J. Kong, and H. Dai, Appl. Phys. Lett. 75, 627 (1999); C. Zhou, J. Kong, and H. Dai, ibid. 76, 1597 (2000).

${ }^{3}$ R. Martel, V. Derycke, C. Lavoie, J. Appenzeller, K. K. Chan, J. Tersoff, and Ph. Avouris, Phys. Rev. Lett. 87, 256805 (2001); Ph. Avouris, R. Martel, V. Derycke, and J. Appenzeller, Physica B 323, 6 (2002).

${ }^{4}$ A. Rochefort, M. Di Vientra, and P. Avouris, Appl. Phys. Lett. 78, 2521 (2001).

${ }_{5}^{5}$ S. Heinze, J. Tersoff, R. Martel, V. Derycke, J. Appenzeller, and Ph. Avouris, Phys. Rev. Lett. 89, 106801 (2002).

${ }^{6}$ M. C. Payne, M. P. Teter, D. C. Allen, T. A. Arias, and J. D. Joannopoulos, Rev. Mod. Phys. 64, 1045 (1992).

${ }^{7}$ J. P. Perdew and Y. Wang, Phys. Rev. B 46, 6671 (1992).

${ }^{8}$ E. Durgun, S. Dag, V. K. Bagci, O. Gülseren, T. Yildirim, and S. Ciraci, Phys. Rev. B 67, 201401(R) (2003).

${ }^{9}$ C. Kilic, S. Ciraci, O. Gülseren, and T. Yildirim, Phys. Rev. B 62, 16345 (2000).

${ }^{10}$ O. Gülseren, T. Yildirim, and S. Ciraci, Phys. Rev. Lett. 87, 116802 (2001).

${ }^{11}$ O. Gülseren, T. Yildirim, S. Ciraci, and C. Kilic, Phys. Rev. B 65, 155410 (2002); O. Gülseren, T. Yildirim, and S. Ciraci, ibid. 65, 153405 (2002).

${ }^{12}$ M. Luna, P. J. de Pablo, J. Colchero, J. Gomez-Herrero, A. M. Baro, H. Tokumoto, and S. P. Jarvis, Ultramicroscopy 96, 83 (2003).

${ }^{13}$ E. Tekman and S. Ciraci, Phys. Rev. B 43, 7145 (1991); S. Ciraci, in Scanning Tunneling Microscopy III, edited by R. Wiesendanger and H.-J. Güntherodt (Springer, Berlin, 1993). 